“ (C) 2018 IEEE. Personal use of this material is permitted. Permission from IEEE must be obtained for all other uses, in any current or future media, including

reprinting/republishing this material for advertising or promotional purposes, creating new collective works, for resale or redistribution to servers or lists, or reuse of any copyrighted component of this work in other works." 


\title{
Reconfigurable, Wideband, Low-Profile, Circularly Polarized Antenna and Array Enabled by an Artificial Magnetic Conductor Ground
}

\author{
Wei Lin, Shu-Lin Chen, Richard W. Ziolkowski, and Y. Jay Guo
}

\begin{abstract}
A reconfigurable, wideband, and low profile circular polarization (CP) antenna is presented. Its wideband $C P$ reconfigurability is realized by incorporating $\mathrm{RF}$ switches in a cross-bowtie radiator. $\mathrm{A}$ compact, wide bandwidth, and polarization independent artificial magnetic conductor (AMC) ground plane is developed to minimize the overall profile of the antenna while maintaining its wide bandwidth. The simplicity of this single element design facilitates the realization of a reconfigurable, wide bandwidth $\mathrm{CP}$ array that achieves higher directivity without changing its overall profile. Prototypes of the single element and of a $1 \times 4$ array of these elements were fabricated and tested. The measured results for both prototypes are in good agreement with their simulated values, validating their design principles. They are low profile with a height $\sim 0.05 \lambda_{0}$. The array exhibits a wide fractional operational bandwidth: $1.65 \mathrm{GHz}(21.7 \%)$, and a high realized gain: $13 \mathrm{dBic}$. Since they would enhance their channel capacity and avoid polarization mismatch issues, these reconfigurable $\mathrm{CP}$ antenna systems are very suitable for modern wireless systems.
\end{abstract}

Index Terms - Artificial magnetic conductor (AMC), circular polarization, crossed dipoles, PIN diodes, reconfigurable antennas, widebandwidth

\section{INTRODUCTION}

Reconfigurable antennas have become increasingly more popular because they provide multi-functional characteristics, including variable frequencies, patterns, and polarizations in compact, light-weight, and cost-effective packages [1] - [7]. Reconfigurable circular polarization (CP) systems, i.e., those that can switch between left-handed (LHCP) and right-handed (RHCP) CP states, offer several unique and highly desirable characteristics. This reconfigurability facilitates the enhancement of the system capacity [8], avoids polarization mismatch [9], and enables polarization coding for wireless systems [10]. Fig. 1 illustrates such capacity enhancement in a satellite communication system. Thanks to the orthogonality of the two CP states, two separate channels can operate in the same frequency band for distinctive applications. For example, the LHCP carrier signal at $f_{0}$ could handle positioning or data transfer tasks while the RHCP carrier signal at the same frequency could be used for telecommunications. Thus, the system's capabilities are doubled. Furthermore, it is acknowledged that the operating bandwidth of the system is another key factor that determines the system capacity. Consequently, CP reconfigurable antennas with wide bandwidth and high directivity are desirable for these aforementioned applications and many others.

Many efforts have been made to realize CP reconfigurability by either adopting switchable feed networks [11] - [15] or designing reconfigurable radiators [16] - [22]. However, the critical challenge has been the associated narrow operating bandwidths. The widest realized $\mathrm{CP}$-operation fractional bandwidth among the above designs is only $7 \%$. The fundamental bandwidth limitations of these antennas are associated with the inherent narrowband characteristics of both the

Manuscript received on Oct. 08, 2017, revision submitted on Dec. 04, 2017

This work was supported in part by the Australian Research Council grant number DP160102219. (Corresponding author: Shu-Lin Chen.)

Wei Lin, Shu-Lin Chen, Richard W. Ziolkowski and Y. Jay Guo are with the University of Technology Sydney, Global Big Data Technologies Centre, Ultimo NSW 2007, Australia (E-mail: wei.lin@uts.edu.au).

Richard W. Ziolkowski is also with the Department of Electrical and Computer Engineering, University of Arizona, Tucson, AZ 85721, USA.

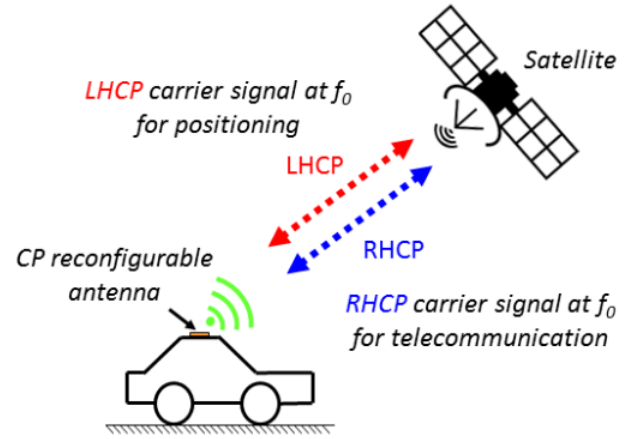

Fig. 1. Reconfigurable CP antenna application for a satellite system. The orthogonal CP states expedite a doubling of the system's capabilities.

radiating elements and feed networks. Although the designs developed in [23] - [25] have significant usable operating bandwidth, up to $23.5 \%$, their structures are complex and would be difficult to extend to antenna array configurations needed to achieve the high directivities required in satellite communication applications.

On the other hand, several reconfigurable antenna array designs have been reported [26] - [29] that do produce high directivities for long distance communications. However, those reported designs are supported only by simulation results due to their implementation difficulties. To the best of our knowledge, only the design reported in [30], a $1 \times 4$ CP polarization reconfigurable antenna array, has been implemented and validated. Reconfigurability was accomplished by introducing RF MEMS switches on four E-shaped patch radiators. The measured CP-operation fractional bandwidth was $20 \%$ and the peak realized gain was approximately $12.8 \mathrm{dBic}$. However, the design has a high fabrication complexity, mainly due to the bonding wires required for its MEMS switches. Moreover, to achieve the reported uni-directional radiation pattern, this patch array required a metallic reflector to be located at $0.09 \lambda_{0}$ below the radiators, where $\lambda_{0}$ is the free-space wavelength of its lowest operating frequency. In practical applications, a lower profile design is preferred.

In this paper, we report the designs, simulations, and measurements of a $\mathrm{CP}$ reconfigurable antenna array that achieves wide $\mathrm{CP}$-operation fractional bandwidth $(21.7 \%)$, is low-profile $\left(0.05 \lambda_{0}\right)$, has high uni-directional realized gain $(13 \mathrm{dBic})$, and has low fabrication complexity. Three innovative contributions are made to obtain these performance characteristics. First, a switchable cross-bowtie radiator is conceived that realizes wideband $\mathrm{CP}$ reconfigurability. Second, a compact, wideband, and polarization-independent AMC reflector is developed to minimize the profile of the antenna to $0.05 \lambda_{0}$. Third, these components are combined successfully with an efficient feed network to realize a reconfigurable, low-profile CP antenna array. Furthermore, the simplicity of the design also signifies that it can be manufactured at low cost.

This paper is organized as follows. The design, operating principles, and experimental verification of the reconfigurable, wideband $\mathrm{CP}$ cross-bowtie antenna integrated with the polarization-independent AMC ground plane are presented in Section II. Four of these elements are then combined in Section III into a $1 \times 4$ configuration to realize the 
reconfigurable, high directivity $\mathrm{CP}$ antenna array. Prototypes of both systems were fabricated and tested. The measured results are shown to be in good agreement with their simulated values. Finally, conclusions are discussed in Section IV.

\section{Reconfigurable, Wideband, Low-Profile CP Antenna}

The AMC structure was developed to have a wide bandwidth and to be polarization independent. By integrating it with a reconfigurable cross-bowie antenna, the low-profile $\mathrm{CP}$ radiating system is obtained. The tested prototype confirms its performance characteristics.

\section{A. AMC Design}

As is well known, a metallic reflector produces a $180^{\circ}$ reflection phase. Consequently, the reflector should be placed a distance of around $0.25 \lambda_{0}$ from the radiator to remove its back lobe and, hence, to realize higher directivity by producing a uni-directional pattern [31]. In contrast, an $\mathrm{AMC}$ reflector produces a $0^{\circ}$ reflection phase. Thus, it can be placed close to the radiator and produce the same effect, yielding a low profile design.

The unit cell of the AMC structure is shown in Fig. 2 (a). It is labeled as AMC-A. While the design was inspired from the AMC unit cell in [32], denoted here as AMC-B, it has a more compact size. The substrates for both are the same with $\varepsilon_{r}=4.4, \mu_{r}=1.0, \tan \delta=0.02$, and thickness $h=8.0 \mathrm{~mm}$. AMC-B consists of a slot-loaded patch layer printed on top of the substrate, and a ground plane on its bottom. The square patch in its center is surrounded by four symmetrical patches. In contrast, $\mathrm{AMC}-\mathrm{A}$ is obtained by etching a square slot in that center patch and connecting it to the ground plane with a shorting post whose radius $r=0.65 \mathrm{~mm}$. Furthermore, the open triangles on the diagonals of AMC-B are filled in symmetrically with quarter pieces of its own design. The final optimized values of AMC-A's design parameters in millimeters were: $L_{s}=58.0, L_{1}=8.0, W_{1}=2.0, L_{2}=20.0, W_{2}=1.0, L_{3}=$ $40.0, W_{3}=2.0$, and $W_{4}=1.0$.

The simulated reflection phases of AMC-A and AMC-B are compared in Fig. 2 (b). The $\pm 90^{\circ}$ phase reflection region for AMC-A yields a $25 \%$ bandwidth centered at $1.68 \mathrm{GHz}$. On the other hand, AMC-B achieves a $24.3 \%$ bandwidth centered at the higher frequency, $2.14 \mathrm{GHz}$. These results indicate that the working frequency band of AMC-A is red-shifted towards lower frequencies with respect to that of AMC-B. Thus, the AMC-A unit cell size per wavelength is $20 \%$ smaller than that of AMC-B, i.e., it is a more compact structure. The reflection phases for AMC-A were studied by scattering a variety of LP waves from it that were incident on it from many different angles. The same reflection phase curves were obtained in all cases, thus confirming its scattering characteristics are polarization independent.

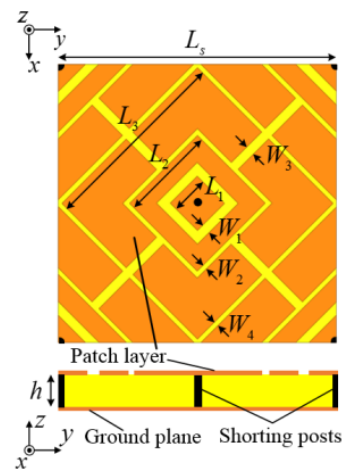

(a)

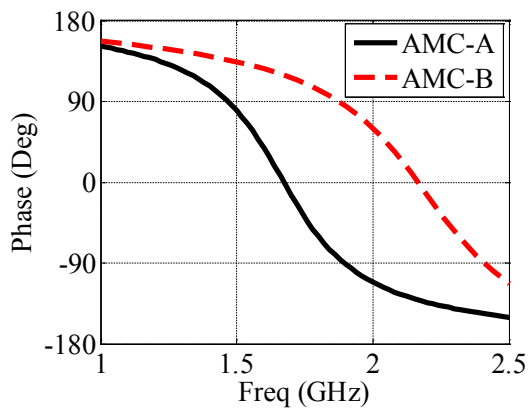

(b)
Fig. 2(a) Unit cell of the AMC reflector design; and (b) Simulated reflection phases from the AMC unit cell in this design and the unit cell in [32].
The reasons for the frequency shift were explored using the parallel LC circuit model introduced in [33]. The resonant frequency is $\omega=$ $1 / \sqrt{L C}$, where $L$ and $C$ are the effective capacitance and inductance of the AMC unit cell. By filling in the four corners of AMC-B, the gaps between neighboring metal pieces are reduced in size and, hence, the effective capacitance is increased. This effect lowers the resonance frequency. The strong couplings associated with these gaps produce multiple resonances. Thus, a wide operating bandwidth is achieved with this gap-coupled structure [32]. The gap widths can be optimized to shift the operating frequencies to desired ranges. To illustrate this effect, the gap width $\mathrm{W}_{3}$ was studied. When $\mathrm{W}_{3}$ is reduced from 4.0 to $2.0 \mathrm{~mm}$, the operating bandwidth remains around $25.0 \%$. On the other hand, the center frequency of the operational range red shifts from 1.76 to $1.68 \mathrm{GHz}$.

\section{B. Integrated Radiating System}

The reconfigurable, wideband $\mathrm{CP}$ cross-bowtie antenna concept originates from related designs given in [34] - [36]. By introducing PIN diodes as RF switches on the cross-bowtie radiator with the proper DC biasing lines, wideband CP reconfigurability was obtained. Due to the integrated AMC reflector, the overall height of the antenna is only $0.05 \lambda_{0}$, which is five times lower than any case with a metallic reflector $\left(0.25 \lambda_{0}\right)$.

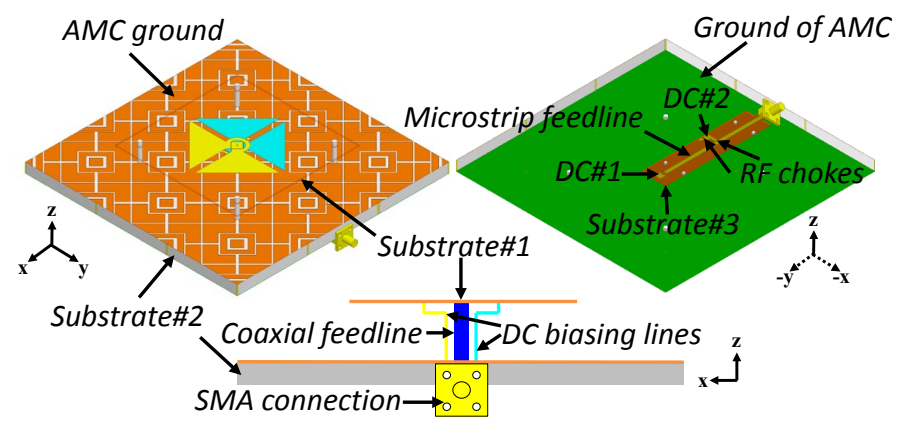

(a)

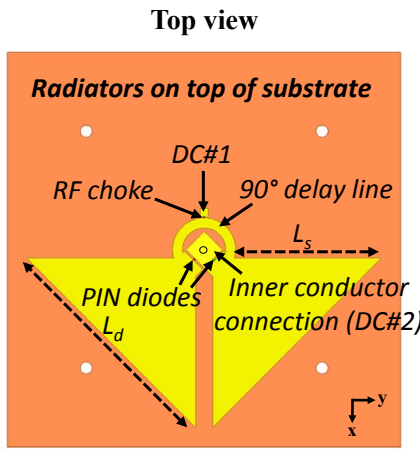

Bottom view

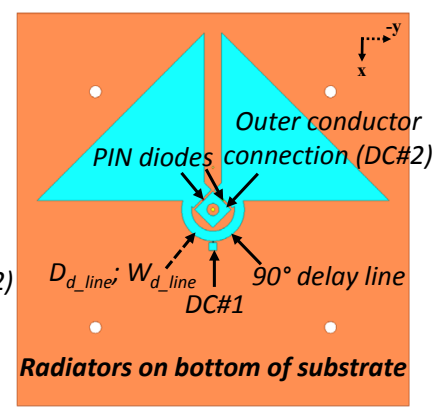

(b)

Fig. 3. Wideband CP reconfigurable cross-bowtie antenna configuration. (a) Perspective and side views. (b) Top and bottom views of the cross-bowtie radiators.

The integrated antenna configuration is shown in Fig. 3. Fig. 3 (a) gives the side and perspective views of the antenna. It consists of three square substrates. The switchable cross-bowtie radiators are printed on the top and bottom layers of the top one, Substrate\#1. The AMC ground is printed on the middle one, Substrate\#2. The microstrip feedline is realized on the bottom one, Substrate\#3. Both Substrate\#1 and \#3 are made from Rogers/Duroid ${ }^{\mathrm{TM}} 5880$ copper-cladded sheets whose $\varepsilon_{r}, \mu_{r}, \tan \delta$, and thickness were 2.2, 1.0, 0.0009, and $0.79 \mathrm{~mm}$, respectively. Substrate\#2 is made from a Wangling Ltd copper-cladded sheet with $\varepsilon_{r}=4.4, \mu_{r}=1.0, \tan \delta=0.02$, and $8.0 \mathrm{~mm}$ 
thickness. The design also includes a piece of coaxial feedline, two DC biasing lines that run along the coax from the bottom side to the diode, four PIN diodes, and six inductors as RF chokes. Four plastic supporting posts are used to provide mechanical support for the structure.

Fig. 3 (b) shows the details of the cross-bowtie radiator. Two orthogonally-oriented triangular elements are printed on the top surface of Substrate\#1 (marked in yellow), and the other pair is located on its bottom surface (marked in blue). The triangular elements in each pair are connected by a short conductive strip, which provides the requisite $90^{\circ}$ phase difference for realizing $\mathrm{CP}$ radiation. These top and bottom pairs are mirror images of each other; they are fed directly by the coaxial cable. In particular, the top two elements are connected to the inner conductor of the coax cable and the bottom two are connected to its outer conductor. Reconfigurability is realized by introducing four PIN diodes that are connected between the bowtie radiators and their center square excitation pads.

The AMC unit cells are located on top of Substrate\#2 and $0.05 \lambda_{0}$ below Substrate\#1. The microstrip feedline is printed on the bottom layer of Substrate\#3. The copper bottom of Substrate\#2 acts as the ground for both the AMC layer and for the microstrip feedline. The feedline is driven with a SMA connector, which produces the coax-to-microstrip transition. The optimized antenna parameters are listed in Table I.

TABLE I

DESIGNPARAMETERS OF THE WIDEBAND CP RECONFIGURABLE ANTENNA WITH THE AMC GROUND (DIMENSIONS IN MILLIMETERS)

\begin{tabular}{clc}
\hline \hline \multirow{2}{*}{ Parameter } & \multicolumn{1}{c}{ Description } & Value \\
\hline$L_{s}$ & Side length of the cross-bowtie radiator & 29.8 \\
$L_{d}$ & Diagonal length of the bowtie radiator & 49.2 \\
$L_{\text {substrate\#1 }}$ & Side length of substrate\#1 & 100.0 \\
$L_{\text {substrate\#2 }}$ & Side length of substrate\#2 & 174.0 \\
$D_{\text {d_line }}$ & Outer diameter of the $90^{\circ}$ delay line & 15.0 \\
$W_{d \_ \text {_line }}$ & Width of the $90^{\circ}$ delay line & 2.5 \\
$d_{\text {gap }}$ & Distance between the bowtie radiators & 4.4 \\
$H$ & Overall height of the antenna & 20 \\
$W_{\text {feedline }}$ & Width of the microstrip feedline & 2.6 \\
$G_{\text {inductor }}$ & Gap for the RF choke inductor & 0.5 \\
$G_{\text {diode }}$ & Gap for the PIN diodes & 0.4 \\
\hline \hline
\end{tabular}

In order to provide the DC biases needed to control the diodes, two DC lines are deployed that provide two bias voltages DC\#1 and DC\#2. As shown in Fig. 3, all of the bowtie radiators are biased with DC\#1 through two vertical wires positioned along the outer wall of the coax feedline. The center square pads are biased with DC\#2 through the microstirp feedline and its ground. As a result, the operational states of the four PIN diodes are controlled simply by the voltages DC\#1 and DC\#2. The layout of the diodes is more clearly depicted in Fig. 4.
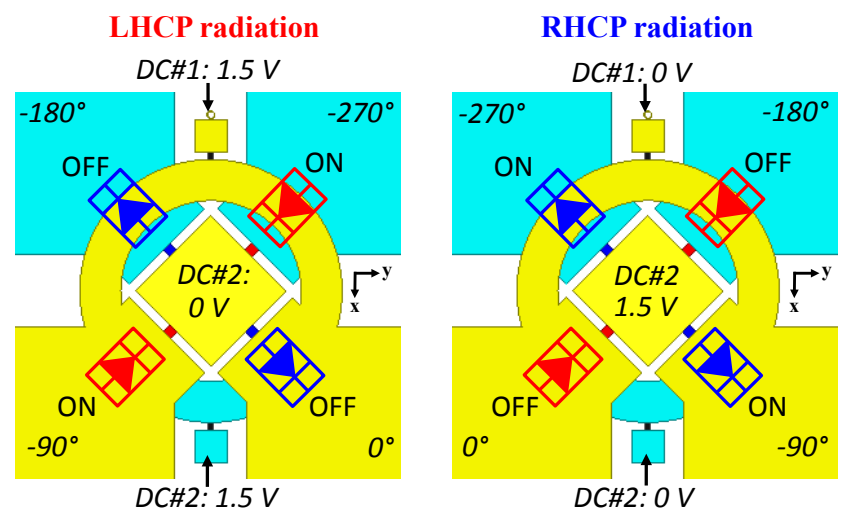

Fig. 4. Diode layout that produces the polarization switching mechanisms.

\section{Operational mechanisms that produce the $C P$ reconfigurability}

One noticeable feature of this design is the simple switching configuration. As Fig. 4 emphasizes, only four PIN diodes are required. This is half the number when compared to the required eight diodes of the wideband CP reconfigurable antenna designs in [24] and [25]. We choose the Bar50-02L PIN diodes from Infineon Technologies with the 0403 surface mount packaging [37]. They have excellent switching performance within our targeted frequency range, L-band from 1 to 2 $\mathrm{GHz}$. The equivalent circuit model and measured characteristics are reported in previous works [38], [39]. The inductors (LQW18AN56NJ00) with 0603 packaging from Murata [40] were selected for the RF chokes.

From the arrangement of the diodes shown in Fig. 4, it is observed that LHCP radiation, for example, will be generated if DC\#1 and $\mathrm{DC} \# 2$ are biased with $1.5 \mathrm{~V}$ and $0 \mathrm{~V}$, respectively. In this state, the two blue diodes aligned along the +45 degree diagonal are turned on and the other two red diodes are off. As a result, the phases of the currents on the four triangular radiators shown in Fig. 4 (LHCP) are $0^{\circ},-90^{\circ}$, $-180^{\circ}$, and $-270^{\circ}$, sequentially delayed in a clockwise manner from the bottom right corner to the top right corner. This clockwise behavior generates the LHCP radiation in the broadside direction. On the other hand, as summarized in Table II, the broadside RHCP radiation mode will be switched on simply by altering the two DC biases.

TABLE II

DC Bias Voltages that Control the Diode and Polarization States

\begin{tabular}{c|c|c|c|c}
\hline \hline DC\#1 & DC\#2 & Red diodes & Blue diodes & Polarization \\
\hline $1.5 \mathrm{~V}$ & $0 \mathrm{~V}$ & ON & OFF & LHCP \\
$0 \mathrm{~V}$ & $1.5 \mathrm{~V}$ & OFF & ON & RHCP \\
\hline \hline
\end{tabular}

\section{Fabricated prototype antenna and measured results}

The antenna components were fabricated, assembled, and tested. A photo of the resulting prototype is given in Fig. 5. It is clearly obsevered that the antenna radiator is close to the AMC ground. Fig. 6 (a) shows the measured and simulated $\left|\mathrm{S}_{11}\right|$ and $\mathrm{AR}$ values as functions of the source frequency. The measured and simulated results are in good agreement. The measured $-10 \mathrm{~dB}$ impedance bandwidth is from 1.24 to $2.0 \mathrm{GHz}(\mathrm{BW}=760 \mathrm{MHz})$. This wideband characteristic results from the bowtie radiators; they support multiple fundamental mode resonances. The bow-tie radiators can be recognized as a truncated, travelling-wave bi-conical antenna. When an ideal, infinitely long travelling-wave antenna is truncated, multi-resonant mode characteristics are attained [41]. Note that a bowtie radiator can be rescaled to other frequency bands by adjusting its size (side and diagonal lengths) while maintaining its tapering angle [31]. The CP-operation fractional bandwidth (overlapped $\left|\mathrm{S}_{11}\right|$ and $\mathrm{AR}$ bandwidths) is $25 \%$, from 1.46 to $1.88 \mathrm{GHz}(\mathrm{BW}=420 \mathrm{MHz})$. This bandwidth is the widest amongst the many previously reported reconfigurable CP designs [11] - [25].

Fig. 6 (b) presents the measured and simulated realized gain values as functions of the source frequency. The measured and simulated results are again in good agreement. It is observed that the co-pol gain values are stable across the entire operational bandwidth with a variation that is less than $3.0 \mathrm{~dB}$. The peak measured realized gain was $6.6 \mathrm{dBic}$. The average co-pol realized gain is $6.1 \mathrm{dBic}(5.8 \mathrm{dBic})$ and the average $\mathrm{x}$-pol realized gain is $-14.8 \mathrm{dBic}(-14.8 \mathrm{dBic})$ for the LHCP (RHCP) mode within the operating bandwidth. Moreover, the measured antenna efficiency is from $65 \%$ to $73 \%$ within that range. 


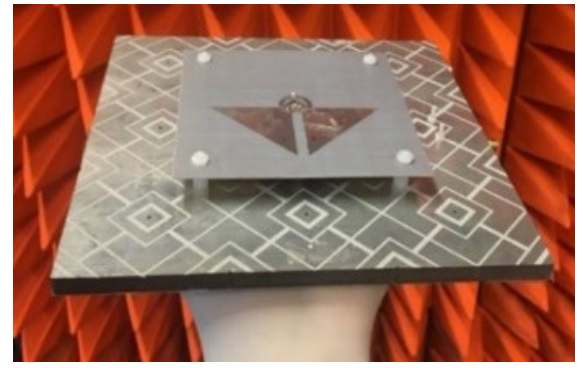

Fig. 5. Fabricated prototype of the wideband CP reconfigurable antenna with the AMC ground.

The corresponding measured normalized realized gain patterns in the two vertical planes: $\varphi=0^{\circ}$ and $\varphi=90^{\circ}$, are presented in Fig. 7. The patterns are given at the center freuqency, $1.65 \mathrm{GHz}$. It is observed that the desired uni-directional broadside radiation was realized. The front-to-back ratio (FTBR) is larger than $25 \mathrm{~dB}$.

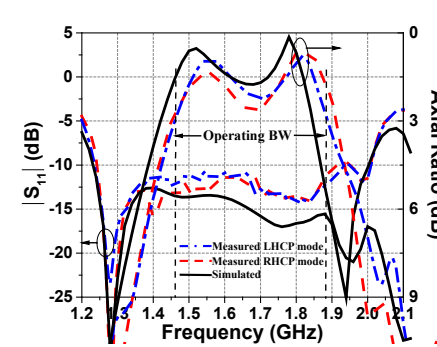

(a)

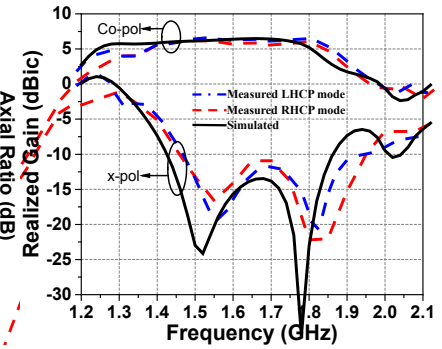

(b)
Fig. 6. Measured and simulated (a) $\left|S_{11}\right|$ and AR values; and (b) realized gain values in both polarization states of the wideband $\mathrm{CP}$ reconfigurable antenna with AMC ground.

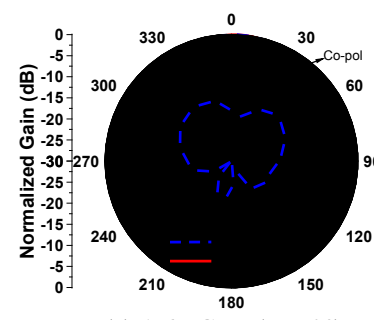

(c) $1.65 \mathrm{GHz}\left(\varphi=0^{\circ}\right)$

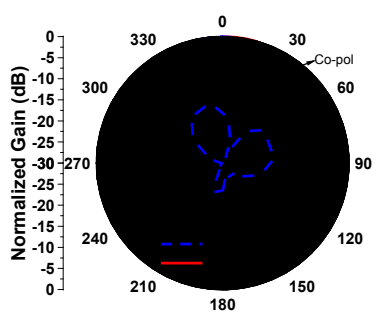

(d) $1.65 \mathrm{GHz}\left(\varphi=90^{\circ}\right)$
Fig. 7. Measured normalized realized gain patterns in the two vertical planes, $\varphi$ $=0^{\circ}$ and $\varphi=90^{\circ}$, of the wideband CP reconfigurable antenna with AMC ground at its center frequency, $1.65 \mathrm{GHz}$, for both of its $\mathrm{CP}$ states.

\section{Wideband Low-Profile CP ReCONFIGURABLE ARRAY}

The simplicity of the CP reconfigurable antenna with AMC ground lends itself to integration into an array with only minimal retuning of its design parameters. The design, fabrication, and testing of the $1 \times 4$ array prototype was completed successfully. The measured performance characteristics were in good agreement with their simulated values.

\section{A. $1 \times 4$ reconfigurable $C P$ array configuration}

The simulation model of the $1 \times 4 \mathrm{CP}$ reconfigurable array is shown in Fig. 8. Four radiating elements were positioned along the $\mathrm{x}$-axis each separated by a distance $d_{s}$. The design parameter dimensions of each single radiating element are the same as the prototype discussed in Section II. There-optimized design parameters are listed in Table III. All other parameters are the same as those of the single element design. A 1-to-4 Wilkinson power divider was developed to feed these four radiating elements. The simple layout of the DC bias lines is shown in the bottom view of the array in Fig. 8. Like the single element design, the polarization state of the array is switched simply by changing the two bias voltages DC\#1 and DC\#2.

Top view

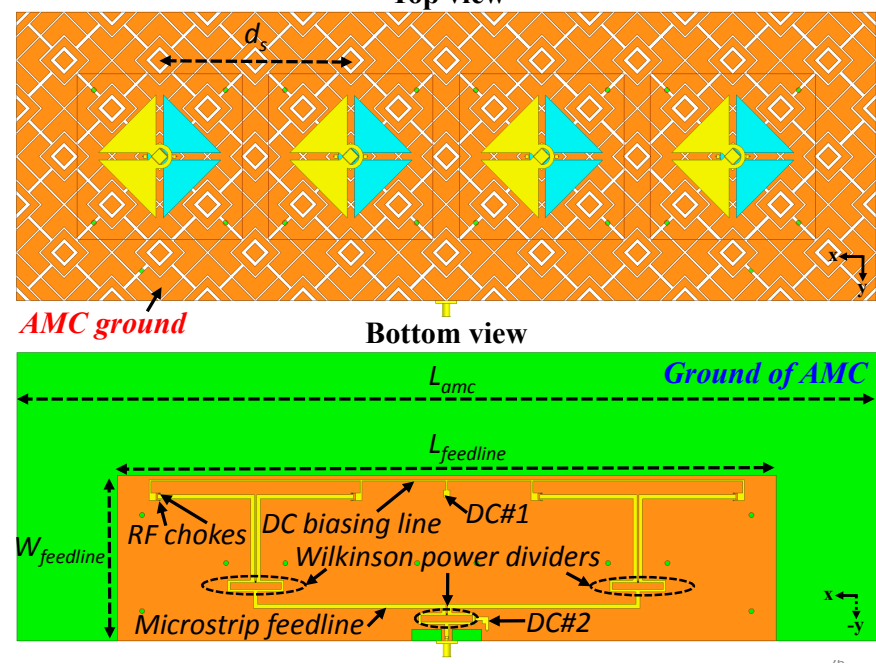

Fig. 8. Simulation model of the $1 \times 4 \mathrm{CP}$ reconfigurable antenna array.

TABLE III

RETUNED DESIGN PARAMETERS THAT OPTIMIZE THE $1 \times 4$ CP RECONFIGURABLE ARRAY (DIMENSIONS IN MILLIMETERS)

\begin{tabular}{clc}
\hline \hline \multirow{2}{*}{ Parameter } & \multicolumn{1}{c}{ Description } & Value \\
\hline$L_{\text {amc }}$ & Length of the AMC structure & 522.0 \\
$L_{\text {feedline }}$ & Length of the microstrip feedline & 400.0 \\
$W_{\text {feedline }}$ & Width of the microstrip feedline & 100.0 \\
$d_{s}$ & Distance between the element centers & 116.0 \\
\hline \hline
\end{tabular}

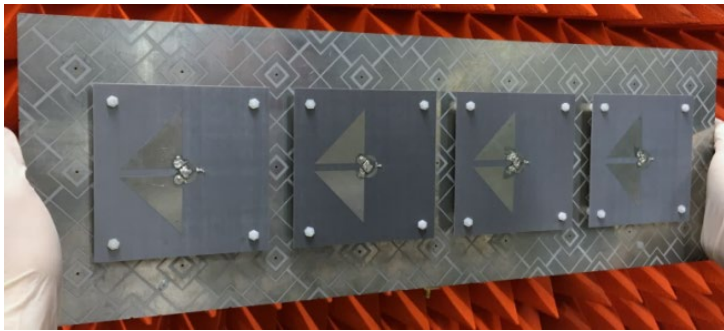

Fig. 9. Fabricated prototype of the $1 \times 4 \mathrm{CP}$ reconfigurable antenna array

\section{B. Fabricated array prototype and measured results}

The optimized $1 \times 4 \mathrm{CP}$ reconfigurable array was fabricated, assembled, and tested. A photo of this prototype is shown in Fig. 9. The overall profile of the array is only $0.05 \lambda_{0}$. This height is $44 \%$ lower than the reconfigurable $\mathrm{CP}$ array design in [30], while also being simpler to fabricate. Fig.10 (a) presents its measured and simulated $\left|\mathrm{S}_{11}\right|$ and AR values as functions of the source frequency. Again, the measured and simulated results are in good agreement. The measured CP-operation fractional bandwidth is $21.7 \%$, from 1.44 to $1.79 \mathrm{GHz}$ $(\mathrm{BW}=350 \mathrm{MHz})$. This array value is slightly smaller than that of the single radiating element $(25 \%)$, but still represents a wide bandwidth.

Fig. 10 (b) shows the measured and simulated realized gain values as functions of the source frequency. Good agreement between measured and simulated results is observed. The realized gain values are stable cross the entire operating bandwidth with a peak value of $13.0 \mathrm{dBic}$. The co-pol. gain variation within the operating bandwidth is less than $3 \mathrm{~dB}$. The corresponding average co-pol realized gain is 11.8 dBic (11.6 dBic) for the LHCP (RHCP) mode and the average $x$-pol realized gain is $-6.3 \mathrm{dBic}(-6.2 \mathrm{dBic})$ for the LHCP (RHCP) mode. The 
measured peak realized gain value of the four-element array is $6.4 \mathrm{~dB}$ higher than that of the single radiating element and slightly exceeds the theoretical value of $6 \mathrm{~dB}$. From additional simulation studies it was determined that because the array was optimized to maximize the realized gain, this gain increase was the trade-off with the slight decrease in the realized bandwidth.

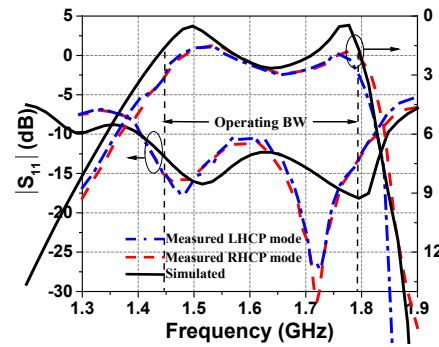

(a)

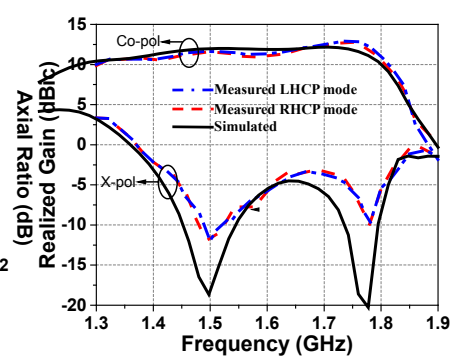

(b)
Fig. 10. Measured and simulated (a) $\left|S_{11}\right|$ and AR values; and (b)realized gain values in both polarization states of the $1 \times 4$ reconfigurable $\mathrm{CP}$ array.

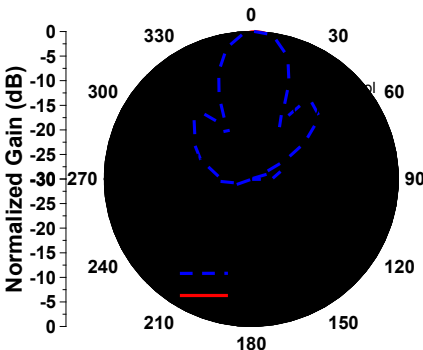

(a) $1.45 \mathrm{GHz}\left(\varphi=0^{\circ}\right)$

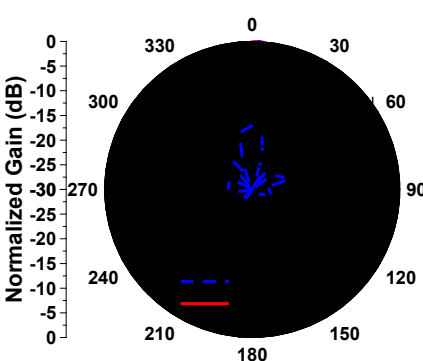

(c) $1.6 \mathrm{GHz}\left(\varphi=0^{\circ}\right)$

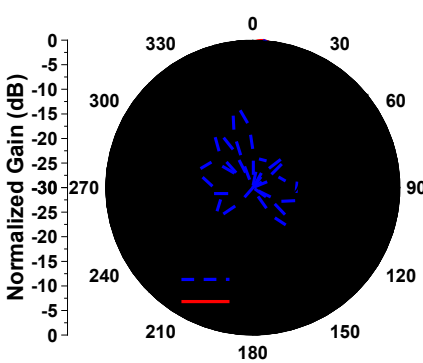

(e) $1.775 \mathrm{GHz}\left(\varphi=0^{\circ}\right)$

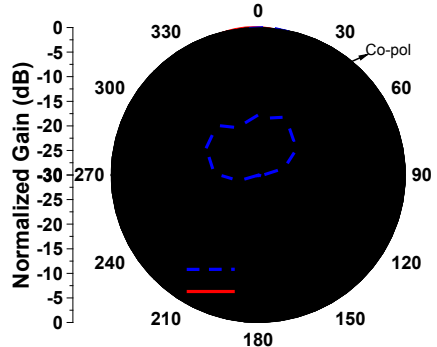

(b) $1.45 \mathrm{GHz}\left(\varphi=90^{\circ}\right)$

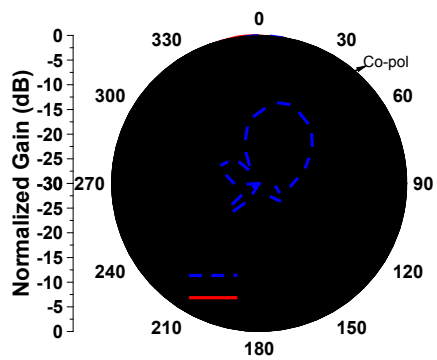

(d) $1.6 \mathrm{GHz}\left(\varphi=90^{\circ}\right)$

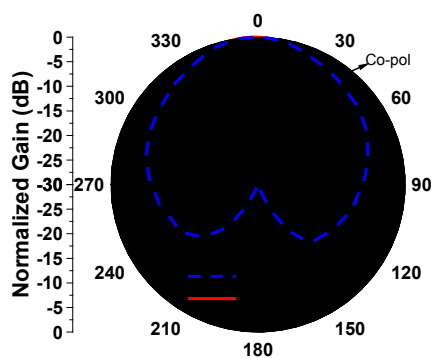

(f) $1.775 \mathrm{GHz}\left(\varphi=90^{\circ}\right)$
Fig. 11. Measured normalized realized gain patterns in the two vertical planes, $\varphi=0^{\circ}$ and $\varphi=90^{\circ}$, of the $1 \times 4 \mathrm{CP}$ reconfigurable array at its center $(1.6 \mathrm{GHz})$ and two bandwidth edge (1.45 and $1775 \mathrm{GHz})$ frequencies for both of its $\mathrm{CP}$ states.

Fig. 11 presents the measured normalized realized gain patterns in the two vertical planes: $\varphi=0^{\circ}$ and $\varphi=90^{\circ}$ at the center frequency (1.6 $\mathrm{GHz}$ ) and the two edge frequencies, $1.45 \mathrm{GHz}$ and $1.775 \mathrm{GHz}$. The desired uni-directional radiation patterns were clearly realized across the entire CP-operation bandwidth. The patterns in the $\varphi=0^{\circ}$ plane have narrower half power beamwidths (HPBW), i.e., $24^{\circ}$ at $1.45 \mathrm{GHz}$, $22^{\circ}$ at $1.6 \mathrm{GHz}$ and $17^{\circ}$ at $1.775 \mathrm{GHz}$, respectively, because of the length of the array along the $x$-axis, which relatively increases as the wavelength (frequency) decreases (increases). In contrast, the patterns in the $\varphi=90^{\circ}$ plane have broad HPBW values as expected, corresponding to the fact that only a single element is along the $x$-axis. The HPBW values are $75^{\circ}$ at $1.45 \mathrm{GHz}, 73^{\circ}$ at $1.6 \mathrm{GHz}$ and $69^{\circ}$ at $1.775 \mathrm{GHz}$, respectively. This frequency-dependent beam width behavior is caused simply by the frequency-dependent effective aperture size. The measured antenna efficiency is from $65 \%$ to $77 \%$ within the operating bandwidth.

As shown by Table IV, the presented experimental results verify, in agreement with their simulated values, that the reconfigurable $\mathrm{CP}$ antenna and the $1 \times 4$ reconfigurable $\mathrm{CP}$ array reported in this paper have good performance characteristics when compared to other reported designs. Our antennas are listed in the table as Ant.\#1, the reconfigurable, wideband, low-profile $\mathrm{CP}$ antenna; and Ant\#2, the corresponding wideband low-profile $1 \times 4$ reconfigurable $\mathrm{CP}$ antenna array. The CP-operation fractional bandwidth, $25 \%$, of Ant.\#1 is the widest amongst similar single element systems. In addition, the height of Ant.\#2 is nearly half that of the array in [30], while having a wider bandwidth and a larger peak realized gain.

TAIVE IV

COMPARISONS OF THE EXPERIMENTALLY VALIDATED CP RECONFIGURABLE ANTENNAS AND ARRAY AND THE REPORTED IN THE LITERATURE

\begin{tabular}{ccccc}
\hline \hline Ref. & No. of elements & $\begin{array}{c}\text { Usable CP } \\
\text { FBW (\%) }\end{array}$ & $\begin{array}{c}\text { Peak gain } \\
(\mathbf{d B i c})\end{array}$ & $\begin{array}{c}\text { Profile } \\
\left(\boldsymbol{\lambda}_{\boldsymbol{\theta}}\right)\end{array}$ \\
\hline$[11]$ & Single & 3.6 & 8.2 & 0.25 \\
{$[12]$} & Single & 4.7 & 2.4 & 0.01 \\
{$[16]$} & Single & 2.8 & 7.5 & 0.06 \\
{$[17]$} & Single & 0.72 & 5.9 & 0.01 \\
{$[18]$} & Single & 1.5 & 2.9 & 0.01 \\
{$[19]$} & Single & 7 & 8.7 & 0.08 \\
{$[23]$} & Single & 15.5 & 4.25 & 0.06 \\
{$[24]$} & Single & 23.5 & 4.8 & 0.26 \\
{$[25]$} & Single & 20.8 & 6.9 & 0.1 \\
{$[30]$} & $1 \times 4$ array & 20 & $\sim 12.8$ & 0.09 \\
Ant.\#1 & Single & $\mathbf{2 5}$ & $\mathbf{6 . 6}$ & $\mathbf{0 . 0 5}$ \\
Ant.\#2 & $1 \times 4$ array & $\mathbf{2 1 . 7}$ & $\mathbf{1 3}$ & $\mathbf{0 . 0 5}$ \\
\hline
\end{tabular}

\section{CONCLUSION}

A reconfigurable wideband $\mathrm{CP}$ antenna and a $1 \times 4$ array of those elements were presented. The design of each system was explained, and the fabrication and testing of their prototypes were described. There configurable, wideband CP antenna combined a reconfigurable cross-bowtie radiator, having a simple switching scheme, with a wideband polarization-independent AMC structure. The AMC allowed the reduction of the overall profile of the antenna system to $0.05 \lambda_{0}$, a 5 -times reduction in height when compared to conventional designs employing a metallic reflector. Four of these elements were combined into a $1 \times 4$ array. This reconfigurable, wideband, low-profile $\mathrm{CP}$ antenna was also fabricated and tested successfully. The measured performance characteristics of both systems were in good agreement with their simulated values. The reported $1 \times 4$ reconfigurable $\mathrm{CP}$ array has a low profile, a wide bandwidth, and a high uni-directional realized gain. This reported $\mathrm{CP}$ antenna systems have the potential to positively impact modern wireless systems for satellite and other applications; they facilitate a practical doubling of their capacities and the mitigation of any polarization mismatch issues. 


\section{REFERENCES}

[1] L. Ge and K. M. Luk, "Frequency reconfigurable low-profile circular monopolar patch antenna," IEEE Trans. Antennas Propag., vol. 62, no. 7, pp. 3443-3449, Jul. 2014.

[2] N. Nguyen-Trong, A. Piotrowski, L. Hall, and C. Fumeaux, "A frequency- and polarization-reconfigurable circular cavity antenna," IEEE Antenna Wireless Propag. Lett., vol. 16, pp. 999-1002, 2016.

[3] P.-Y. Qin, Y. J. Guo, Y. Cai, E. Dutkiewicz, and C.-H. Liang, "A reconfigurable antenna with frequency and polarization agility," IEEE Antenna Wireless Propag. Lett., vol. 10, pp. 1373-1376, 2011.

[4] S. V. Hum, M. Okoniewski, and R. J. Davies, "Modeling and design of electronically tunable reflect arrays," IEEE Trans. Antennas Propag., vol. 55, no.8, pp. 2200-2210, Aug. 2007.

[5] J. T. Bernhard, Reconfigurable Antennas, London. UK: Morgan \& Claypool, 2007.

[6] N. Behdad and K. Sarabandi, "Dual-band reconfigurable antenna with a very wide tunability range," IEEE Trans. Antennas Propag., vol. 54, no. 2 , pp. 409-416, Feb. 2006.

[7] J. Y. Deng, S. Hou, L. Zhao, and L. X. Guo, "A Reconfigurable Filtering Antenna with Integrated Band-pass Filters for UWB/WLAN Applications," IEEE Trans. Antennas Propag., accepted. DOI: 10.1109/TAP.2017.2760363.

[8] C. G. Christodoulou, Y. Tawk, S. A. Lane, and S. R. Erwin, "Reconfigurable antennas for wireless and space applications," Proc. IEEE, vol. 100, pp. 2250-2261, Jul. 2012.

[9] H. Wong, W. Lin, L. Huitema, and E. Arnaud, "Multi-polarization reconfigurable antenna for wireless biomedical system," IEEE Trans. Biomed. Circuit Syst., vol. 11, no.3, pp. 652 - 660, Mar. 2017.

[10] M. A. Kossel, R. Kung, H. Benedickter, and W. Biichtokd, "An active tagging system using circular-polarization modulation," IEEE Trans. Microw. Theory Tech., vol. 47, no. 12, pp. 2242-2248, Jun. 1999.

[11] F. Wu and K. M. Luk, "Single-port reconfigurable magneto-electric dipole antenna with quad-polarization diversity," IEEE Trans. Antennas Propag., vol. 65, no. 5, pp. 2289-2296, May 2017.

[12] J.-S. Row, W.-L. Liu, and T.-R. Chen, "Circular polarization and polarization reconfigurable designs for annular slot antennas," IEEE Trans. Antennas Propag., vol. 60, no.12, pp. 5998-6002, Dec. 2012.

[13] H. Aïssat, L. Cirio, M. Grzeskowiak, J.-M. Laheurte, and O. Picon, "Reconfigurable circularly polarized antenna for short-range communication systems," IEEE Trans. Microw. Theory Tech., vol. 54, no. 6, pp. 2856-2863, Jun. 2006.

[14] K. Boonying, C. Phongcharoenpanich, and S. Kosulvit, "Polarization reconfigurable suspended antenna using RF switches and P-I-N diodes," in Proc. The 4th Joint International Conference on Information and Communication Technology Electronic and Electrical Engineering (JICTEE-2014), Chiang Rai, Thailand,Mar. 2014.

[15] H. Sun and S. Sun, "A novel reconfigurable feeding network for quad-polarization-agile antenna design," IEEE Trans. Antennas Propag., vol. 64, no. 1, pp. 311-316, Jan. 2016.

[16] P.-Y. Qin, A. R. Weily, Y. J. Guo, and C.-H. Liang, "Polarization reconfigurable U-slot patch antenna," IEEE Trans. Antennas Propag., vol. 58, no. 10, pp. 3383-3388, Oct. 2010.

[17] B. Kim, B. Pan, S. Nikolaou, Y.-S. Kim, J. Papapolymerou, and M. M. Tentzeris, "A novel single-feed circular microstrip antenna with reconfigurable polarization capability," IEEE Trans. Antennas Propag., vol. 56, no. 3, pp. 630-638, Mar. 2008.

[18] Z.-X. Yang, H.-C. Yang, J.-S. Hong, and Y. Li, "Bandwidth enhancement of a polarization-reconfigurable patch antenna with stair-slots on the ground," IEEE Antennas Wireless Propag. Lett., vol. 13, pp. 579-582, 2014.

[19] A. Khidre, K.-F. Lee, F. Yang, and A. Z. Elsherbeni, "Circular polarization reconfigurable wideband E-shaped patch antenna for wireless applications," IEEE Trans. Antennas Propag., vol. 61, no.2, pp. 960-964, Feb. 2013.

[20] N. H. Noordin, W. Zhou, A. O. El-Rayis, N. Haridas, A. T. Erdogan, and T. Arslan, "Single-feed polarization reconfigurable patch antenna," in Proc. 2012 IEEE Antennas and Propagation Society International Symposium (APSURSI2012), Chicago, IL, Jul.2012.

[21] T. Song, Y. Lee, D. Ga, and J. Choi, "A polarization reconfigurable microstrip patch antenna using PIN diodes," in Proc. 2012 Asian Pacific Microwave Conference(APMC 2012), Kaohsiung, Taiwan, Dec. 2012, pp 616-618.
[22] W. Li, S. Gao, Y. Cai, Q. Luo, M. Sobhy, G. Wei, J. Xu, J. Li, C.Wu, and Z. Cheng, "Polarization-reconfigurable circularly polarized planar antenna using switchable polarizer," IEEE Trans. Antennas Propag., vol. 65, no. 9, pp. 4470-4477, Sep. 2017.

[23] W. Yang, W. Che, H. Jin, W. Feng, and Q. Xue, "A polarization-reconfigurable dipole antenna using polarization rotation AMC structure," IEEE Trans. Antennas Propag., vol. 63, no. 12, pp. 5305-5315, Dec. 2015.

[24] W. Lin and H. Wong, "Wideband circular polarization reconfigurable antenna," IEEE Trans. Antennas Propag., vol. 63, no. 12, pp. 5938-5944, Dec. 2015.

[25] W. Lin and H. Wong, "Wideband circular polarization reconfigurable antenna with L-shaped feeding probes," IEEE Antenna Wireless Propag. Lett., vol. 16, pp. 2114-2117, 2017.

[26] G. A. Wang, T. Polley, A. Hunt, and J. Papapolymerou, "A high performance tunable RF MEMS switch using barium strontium titanate (BST) dielectrics for reconfigurable antennas and phased arrays," IEEE Antenna Wireless Propag. Lett., vol. 4, pp. 217-220, 2005.

[27] E. Nishiyama, M. Aikawa, and S. Sasaki, "Polarisation switchable slot-ring array antenna," IET Microwaves, Antennas \& Propagation, vol. 2, no.3, pp. 236-241, 2008.

[28] D. V. Navarro-Méndez, L. F. Carrera-Suárez, M. Baquero-Escudero, and H. C. Moy-Li, "Reconfigurable array antenna in LTCC technology," in Proc. 2014 IEEE Antennas and Propagation Society International Symposium (APSURSI2014), Memphis, TN, Jul.2014, pp. 1670-1671.

[29] T. Onishi, M. A. Hossain, E. Nishiyama, and I. Toyoda, "Linear polarization switchable microstrip array antenna using Magic-T circuit," in Proc. IEEE International Symposium on Antennas and Propagation (ISAP2012), Nagoya, Japan, Oct.-Nov. 2012, pp. 858-861.

[30] J. M. Kovitz, H. Rajagopalan, and Y. Rahmat-Samii, "Design and implementation of broadband MEMS RHCP/LHCP reconfigurable arrays using rotated E-Shaped patch elements," IEEE Trans. Antennas Propag., vol. 63, no. 6, pp. 2497-2507, Jun. 2015.

[31] C. A. Balanis, Antenna Theory. 3rd ed. New York: John Wiley \& Sons, 2005

[32] H. Malekpoor and S. Jam, "Improved radiation performance of low profile printed slot antenna using wideband planar AMC surface," IEEE Trans. Antennas Propag., vol. 64, no. 11, pp. 4626-4638, Nov. 2016.

[33] D. Sievenpiper, L. Zhang, R. F. J. Broas, N. G. Alexopolous, and E. Yablonovitch, "High-impedance electromagnetic surfaces with a forbidden frequency band," IEEE Trans. Microw. Theory Tech., vol. 47, no. 11, pp. 2059-2074, Nov. 1999.

[34] S. X. Ta, I. Park, and R. W. Ziolkowski, "Crossed dipole antennas: a review.," IEEE Antennas Propagat. Mag., vol. 57, no. 5, pp. 107-122, Oct. 2015.

[35] S. X. Ta, K. Lee, I. Park and R. W. Ziolkowski, "Compact crossed-dipole antennas loaded with near-field resonant parasitic elements," IEEE Trans. Antennas Propag., vol. 63, no. 6, pp. 2497-2507, Jun. 2015.

[36] Y. He, W. He, and H. Wong, "A wideband circularly polarized cross-dipole antenna," IEEE Antenna Wireless Propag. Lett., vol. 13, pp. 67-70, 2014.

[37] Datasheet Bar50-02L PIN Diodes. (2017, Oct. 2). Infineon Technologies, Application Note [Online]. Available: http://www.infineon.com/.

[38] W. Lin and H. Wong, "Polarization reconfigurable aperture-fed patch antenna and array," IEEE Access, vol. 4, pp. 1510-1517, Apr. 2016.

[39] W. Lin and H. Wong, "Polarization reconfigurable wheel-shaped antenna with conical-beam radiation pattern," IEEE Trans. Antennas Propag., vol. 63, no. 2, pp. 491-499, Feb. 2015.

[40] Datasheet LQW18AN56NJ00Inductor. (2017, Oct. 2). Murata Manufacturing Co., Ltd., Application Note [Online]. Available: http:// www.murata.com/

[41] C. H. Papas and R. King, "Input impedance of wide-angle conical antennas fed by a coaxial line," Proc. IRE, vol. 37, pp. 1269-1271, Nov. 1949. 